\title{
BMJ Open Introducing new point-of-care tests for common infections in publicly funded clinics in South Africa: a qualitative study with primary care clinicians
}

Oliver van Hecke, ${ }^{1}$ Chris Butler, ${ }^{1,2}$ Marc Mendelson, ${ }^{3}$ Sarah Tonkin-Crine ${ }^{1,2}$

To cite: van Hecke 0, Butler C, Mendelson M, et al. Introducing new point-of-care tests for common infections in publicly funded clinics in South Africa: a qualitative study with primary care clinicians. BMJ Open 2019;9:e029260. doi:10.1136/ bmjopen-2019-029260

- Prepublication history and additional material for this paper are available online. To view these files, please visit the journal online (http://dx.doi. org/10.1136/bmjopen-2019029260).

Received 22 January 2019 Revised 20 September 2019 Accepted 07 November 2019

Check for updates

(c) Author(s) (or their employer(s)) 2019. Re-use permitted under CC BY-NC. No commercial re-use. See rights and permissions. Published by BMJ.

${ }^{1}$ Nuffield Department of Primary Care Health Sciences, University of Oxford, Oxford, UK ${ }^{2}$ NIHR Health Protection Research Unit in HealthcareAssociated Infections and Antimicrobial Resistance, University of Oxford, Oxford, UK ${ }^{3}$ Division of Infectious Diseases and HIV Medicine, Department of Medicine, Groote Schuur Hospital, University of Cape Town, Cape Town, UK

Correspondence to

Dr Oliver van Hecke; oliver.vanhecke@phc.ox.ac.uk

\section{ABSTRACT}

Broad-spectrum antibiotics are routinely prescribed empirically in the resource-poor settings for suspected acute common infections, which drive antimicrobial resistance. Point-of-care testing (POCT) might increase the appropriateness of decisions about whether and which antibiotic to prescribe, but implementation will be most effective if clinician's perspectives are taken into account. Objectives To explore the perceptions of South African primary care clinicians working in publicly funded clinics about: making antibiotic prescribing decisions for two common infection syndromes (acute cough, urinary tract infection); their experiences of existing POCTs; their perceptions of the barriers and opportunities for introducing (hypothetical) new POCTs.

Design, method, participants, setting Qualitative semistructured interviews with 23 primary care clinicians (nurses and doctors) at publicly funded clinics in the Western Cape Metro district, South Africa. Data were analysed using thematic analysis.

Results Clinicians reported that their antibiotic prescribing decisions were influenced by their clinical assessment, patient comorbidities, social factors (eg, access to care) and perceived patient expectations. Their experiences with currently available POCTs were largely positive, and they were optimistic about the potential for new POCTs to: support evidence-based prescribing decisions that might reduce unnecessary antibiotic prescriptions; reduce the need for further investigations; support effective communication with patients, especially when antibiotics were unlikely to be of benefit. Resources and workflow disruption were seen as the main barriers to uptake into routine care.

Conclusions Clinicians working in publicly funded clinics in the Western Cape Metro of South Africa saw POCTs as potentially useful for positively addressing both clinical and social drivers of the overprescribing of broad-spectrum antibiotics, but were concerned about the resource implications and disruption of existing patient workflows.

\section{BACKGROUND}

Antibiotic resistance is an important societal health issue and is central to the global health policy agenda. Recent antibiotic use in the community is the most important risk factor for a patient developing a subsequent
Strengths and limitations of this study

- This qualitative study with primary care clinicians in publicly funded clinics in South Africa gives insight into their perceptions about making antibiotic prescribing decisions for common infection syndromes and the potential uptake of new point-of-care tests (POCTs).

- We obtained data from both nurse and doctor prescribers to reflect the breadth of antibiotic prescribing practice and because many patients are managed exclusively by nurses.

- Clinicians in this study did not have experience of using new POCTs, and therefore, their views may not reflect how they would feel about tests after having used them in practice.

- While this study reports clinicians' perceptions of how patients may respond to new POCTs, direct study of patients' views is recommended.

resistant bacterial infection. ${ }^{1}$ Patients with antibiotic-resistant infections are sicker for longer and more difficult and expensive to treat. ${ }^{2}$ Most antibiotics are prescribed in the community or ambulatory healthcare settings (general practice, outpatient, emergency departments), and many of these are unnecessary or unnecessarily broad spectrum, which drives antibiotic resistance. ${ }^{3-6}$

The largest increase in antibiotic prescribing over a 10-year period among the BRICS nations (Brazil, Russia, India, China and South Africa) was in South Africa, reflecting the burden of infectious diseases and high rates of drug-resistant bacterial infections, ${ }^{7}$ complicated by the disproportionate impact of HIV and tuberculosis (TB). ${ }^{8}$ The Standard Treatment Guidelines and essential medicines list for South Africa, (Primary Healthcare Level) provides an evidence-based guideline for prescribing in primary care. ${ }^{9}$ However, limited published research and evaluation of antibiotic prescribing in ambulatory care in South Africa exists especially in the public 
sector because of a lack of integrated pharmacy, laboratory and clinical information systems. ${ }^{10-13}$ In a unique point-prevalence survey of antibiotic prescribing in primary care facilities in the Cape Town Metro (2016), less than half of antibiotic prescriptions adhered to national antibiotic primary care guidelines. ${ }^{11}$ Evidence from highincome countries (HICs) suggests that reasons for poor compliance to guidelines for self-limiting respiratory tract infections (RTIs) like acute cough may be related to: concerns about maintaining the doctor-patient relationship; perceived patient expectations for antibiotic treatment; diagnostic uncertainty; clinician's habitual prescribing practice; overestimation of the benefit from antibiotics; or that prescribing is more efficient than discussing non-antibiotic management strategies in timepressured consultations. ${ }^{14-21}$

Although effective interventions to improve community antibiotic prescribing in HIC settings have been identified including point-of-care testing (POCT), ${ }^{22} 23$ these diagnostic tools are generally less available in the resourcepoor settings. ${ }^{24}$ Exploring the potential use of POCTs for common infection syndromes in low-middle-income countries (LMICs) is of particular interest for three reasons: (1) the overuse of especially broad-spectrum antibiotics alongside the higher risk of undertreating potentially severe bacterial infections, for example, TB; (2) laboratory facilities in primary care are often inadequate $^{25}$ and (3) the shortage of a skilled health workforce. However, it is encouraging to see an increasing number of studies from LMICs (in particular southeast Asia) that have evaluated the use of POCTs for common infection syndromes (beyond HIV, sexually transmitted diseases, $\mathrm{TB}$ and malaria). These studies included randomised trials using $\mathrm{C}$ reactive protein (CRP) POCTs to guide prescribing decisions. ${ }^{26-30}$ However, findings from these studies do not necessarily apply to other LMICs where disease spectra and the social and cultural determinants of health and healthcare systems differ. ${ }^{28}{ }^{31}$ Developing and implementing interventions to improve the quality of antibiotic prescribing for common infection syndromes are more likely to be effective if sensitive to understanding prescribers' perceptions of their current practice and their perceptions about context-specific opportunities and challenges for the uptake of new POCTs.

We, therefore, set out to explore the views and experiences of clinicians (nurses and doctors) at publicly funded day hospitals (primary care clinics) in the Western Cape Metro, South Africa about: making antibiotic prescribing decisions for two common infection syndromes (acute cough, urinary tract infection); using existing POCTs; introducing (hypothetical) new POCTsand the likely barriers and opportunities of introducing new POCTs for these common infections in their workplace.

\section{METHODS}

We used qualitative research methods involving narrative and semistructured face-to-face interviews with clinicians across the Western Cape Metro, South Africa. The Cape Metro is a periurban region around Cape Town of just over $290 \mathrm{~km}$. It includes the suburbs of Khayelitsha and Mitchells Plain with an estimated population of over four million. Based on 2011 census data, Mixed race (42\%) and Black African (37\%) population groups predominate in the Cape Metro. ${ }^{32}$ The majority of the population are uninsured $(77 \%)$. Access to basic services such as water, sanitation and electricity is high $(>85 \%)$, unemployment is high $(30 \%)$, with a substantial proportion $(16 \%)$ of households earning < $£ 240$ (R4800) per annum and living in informal dwellings (20.5\%). There are 152 public primary healthcare facilities and 8 district hospitals in the Cape Metro region. Access to primary care health services is free of charge. ${ }^{33}$ Obtaining antibiotics are through prescription only. Antibiotic prescribing data in ambulatory (primary) care are not routinely available.

\section{Participants and setting}

Primary care clinicians were recruited by advertising the study through the Cape Town Family Physician Forum and at a regional nurse managers' workshop at the University of the Western Cape. Additional participants were recruited through snowballing and personal contacts at the Department of Family Medicine, University of Cape Town. We invited clinicians from a variety of settings: nurse-led clinics/day hospitals with contrasting social deprivation, population demographics, clinic size (number of clinicians) and antibiotic prescribing pattern (personal communication, Renier Coetzee). Clinicians included any nurse or medical doctor (general practitioner, family physician) who makes antibiotic prescribing decisions for patients with common infections. Here, we use the term 'common infections' to refer to the two common infection syndromes (acute cough, urinary tract infection).

\section{Interviews}

Interviews followed a topic guide (online supplementary appendix), which included exploratory questions about how the management of common infections could be improved and to gauge clinicians' ideas about a new (hypothetical) POCTs that could support clinical decision-making. Interview questions were informed by studies done in HIC settings, and adapted after advice from clinicians practising in the Western Cape Metro and in response to emerging data. ${ }^{34-40}$ Participants were asked about their experience of existing POCTs, and their opinions of new POCTs for the specified two common infection syndromes. Participants were expressly told that these (new) POCTs would likely be similar to a finger-prick blood test, swab or urine sample that could be performed in the clinic within several minutes. No specific test platforms were mentioned. Interviews were conducted by OvH in English at the participant's clinic, home or other suitable setting of their preference. Written informed consent was obtained prior to interview. Interviews were audio recorded and transcribed verbatim. 


\section{Analysis}

Transcripts were checked for accuracy against the interview recording, anonymised and then analysed using thematic analysis. ${ }^{41}$ Thematic analysis is a systematic method for data analysis, involving six stages, which allows researchers to take an inductive approach. This was important to allow us to identify aspects of the data which were most important to participants which may not have been apparent to the research team a priori. Specialist software (NVivo V.11) was used to organise data and support ease of analysis. Constant comparison was used to compare data across interviews, to identify similarities and differences between participants and contexts, and to identify deviant cases. ${ }^{42}$ We systematically coded meaningful units of text which related to the research questions from the data using a line by line approach. Codes were categorised, grouping similar codes together. Categories were organised into a framework to provide themes and subthemes based on ten coded interviews. A sample of the transcripts $(20 \%)$ was coded by both OvH and ST-C and agreement on themes and subthemes within the framework, was sought. This coding framework was then applied to subsequent transcripts with amendments and additions made as necessary.

\section{Patient and public involvement}

No patients were involved in this study. Clinicians informed the development of the topic guide.

\section{Researcher characteristics and reflexivity}

The principal investigator $(\mathrm{OvH})$ was mindful that his personal, cultural and experiential background as a South African-trained clinician would bring in potential biases and values.

OvH was familiar with the local languages and healthcare systems. This seemed to get more traction from potential participants during recruitment, and participants were responsive, open and appeared to place their trust in a fellow colleague. OvH strove to use an openended, non-judgemental approach to questioning within interviews, letting participants 'tell their story'. Data analysis was carried out with a senior coauthor (ST-C), a social scientist with limited knowledge of South African healthcare, and both authors refined the coding strategy and developed the thematic framework.

\section{RESULTS}

Twenty-three clinicians were interviewed between 1 March and 30 April 2018 from 13 periurban primary care clinics. The majority of participants were female $(n=14)$, with an average age of 42 years. Most were recruited through regional Family Physician Forum contacts. Among 23 clinicians, 8 were nurse prescribers, 4 were doctors without specialisation (medical officers) and 11 were family physicians. Nine clinicians self-identified as Mixed race, nine as White, and five clinicians as Black-African. The number of participants from each clinic ranged
Box 1 Main themes and subthemes

\section{Theme 1 Context of antibiotic prescribing.}

1.1 Carrying out antibiotic guideline-led practice.

1.2 Monitoring of antibiotic prescribing.

1.3 Antibiotic prescribing in the context of serious infections.

1.4 Factors influencing antibiotic prescribing for acute cough, urinary tract infection.

1.5 Diagnosis of common infections and special investigations available.

Theme 2 providing patient care in South Africa

2.1 Constraints of the current South African health system.

2.2 Patient access to care.

2.3 Patient expectations of care provided.

2.4 Explanations of antibiotic use and patient acceptance.

2.5 Safety netting advice and alternatives to antibiotics.

Theme 3 User experiences of point-of-care tests (POCTs) and the potential for new POCTs for common infections

3.1 Perceptions of new POCTs based on past experience.

3.2 Potential use of POCTs in decision-making for common infection syndromes.

3.3 Patient benefits of POCTs and its likely impact on the consultation. 3.4 Cost implications of POCTs.

3.5 Workload of performing, maintaining and storing POCTs.

between one and six participants. Interviews ranged from 22 to $59 \mathrm{~min}$ in duration, with a mean of $38 \mathrm{~min}$. Three main themes were identified to represent the data, illustrated below with quotations (box 1).

\section{Theme 1 context of antibiotic prescribing}

Clinicians were generally positive about national antibiotic prescribing guidelines that use a syndromic approach to guide management of common infections, for example, acute cough. Most felt they were practising according to these guidelines, and reported that they often followed a non-antibiotic management strategy for common infections.

We go quite strictly according to the guidelines unless there [are] good reasons to deviate from them. Then we have to explain to the pharmacist or motivate [the reason for prescribing a specific antibiotic not outlined in the guideline]. (P1, doctor)

Clinicians described antibiotic stewardship initiatives in primary care (eg, prescribing audits) as relatively new to their context. They reported that these audits were more likely to be conducted in larger, urban public and private sector clinics than in rural clinics. A small proportion of participants regarded these prescribing audits as simply adding unhelpfully to workload. Clinicians felt that achieving guideline congruent prescribing was less of a priority than enhancing the management of more serious infections like HIV, TB and rheumatic fever. Clinicians based their management on clinical history and examination findings, but also took comorbidities, other social determinants of health (eg, access to care) and perceived patient expectations for antibiotics into account. 
Sometimes you know it's a viral thing, but because the patient might be elderly, might not have access to the facility, doesn't have transport to come back [...]. So, you sort of soothe your conscience by prescribing prophylactic antibiotics. (P4, doctor)

Clinicians accepted that in the absence of a laboratoryconfirmed infection, clinical diagnosis was 'never 100\%' $(\mathrm{P} 2$, doctor) and there were many 'grey zones' (P13, doctor) where it was unclear whether patients were likely to benefit from antibiotics. They were less confident about making prescribing decisions for common infections when patients were also living with HIV/TB. This often led to prescribing empiric antibiotics even when existing POCTs (eg, urine dipstick) were negative, or before laboratory test results were available. Clinicians acknowledged that many antibiotics were prescribed unnecessarily for probable self-limiting acute RTIs.

I think that's the biggest struggle in the clinic, you know, we are treating viral upper RTIs as bacterial infections. (P12, doctor)

Clinicians indicated that the unavailability or considerable delay in getting results back from laboratories meant that blood and sputum tests for acute infections hardly ever influenced their antibiotic prescribing decisions. Occasionally, a chest X-ray would be performed on-site to appease patients' expectations for antibiotics.

\section{Theme 2 providing patient care in South Africa}

All clinicians were serving socioeconomically disadvantaged populations with a high burden and prevalence of HIV-TB coinfection. Data about the incidence, clinical features, microbiology and outcomes of acute common infections were not available for their context. Clinics offered a walk-in service for acute problems and booked clinic appointments were generally for patients with chronic disease. Clinicians reported that patients were rarely given follow-up appointments.

So, you don't want the patient to come back again. The clinic is full, and to get the person to come back in 3 days' time to make sure that their upper RTI is not a bacterial tonsillitis. It's just not going to happen, so an antibiotic gets prescribed. (P16, doctor)

Clinicians reported that there were only few antibiotic classes available in their clinics to choose from, and that antibiotics were often out-of-stock. Budget constraints limited special investigations. Although non-antibiotic treatment was available on prescription, cough syrup was not. There was an overall perception of inertia to change healthcare in the public sector.

I think we, we sort of function in an environment where we don't know what we could use and so [...], I find myself quite happy to carry on as I am... (P13, doctor)
Access to healthcare was seen as difficult, timeconsuming and costly. Clinicians reported that many patients made long journeys to get to and from clinics. This together with the sheer volume of patients attending clinics, further limited opportunity for the follow-up. Delayed antibiotic prescribing was, therefore, uncommon. Clinicians reported that there was a small, distinct group of patients that simply consulted to obtain a sick certificate, that some were seeking reassurance from a healthcare professional, and that other patients simply wanted special investigations (eg, chest X-ray) rather than seeking antibiotics. Most clinicians perceived that many patients expected antibiotics when consulting for common infections, either because of previous use for a similar illness, or because a non-antibiotic management strategy was costly (as opposed to antibiotic prescriptions which are free at publicly funded clinics). A few clinicians were confident in managing patient expectations for antibiotics by addressing patients' beliefs about antibiotics and potential harms to help patients understand the reasons for their prescribing decision.

So, you need to be very knowledgeable. If the [patients] find out that you [are] not very sure of yourself especially when you keep on referring to your guidelines they [will not] trust you. (P7, nurse)

However, involving patients in treatment decisions for common infections appeared uncommon. Clinicians reported that patients tended not to voice their dissatisfaction about care they received. Some clinicians perceived that many patients would accept a non-antibiotic management strategy if the clinician took the time to examine them, explain their reasoning and certify time off work for those in employment. However, others thought that patients expected clinicians to 'give' them something (antibiotic) following a consultation. Clinicians perceived that patients would often tend to 'clinic hop' in order to obtain antibiotics.

...You try to explain to [patients] there's a difference between a virus and a bacterium and if you only have the flu $[\ldots]$, antibiotics are not going to make a difference to you and they look at you like you're mad. So, they [say] 'why aren't you doing anything for me, I'm coming to you for help and you're not giving me anything'. (P12, doctor)

Communication difficulties often hampered efforts to explain a non-antibiotic management strategy. Multiple, local African languages (and relative paucity of scientific medical terms) made patient education about the differences between bacterial and viral infections often ineffective and hindered efforts to discuss more complex issues such as antibiotic resistance.

In Xhosa, there is only one word, kwamagciwane [bacteria], which means germs. Even that word, kwamagciwane, now has the connotation of-that's HIV. (P16, doctor) 
Theme 3 user experiences of POCTs and the potential for new POCTs for common infections

All clinicians had experience with existing clinic POCTs, mainly for HIV, syphilis and suspected urinary tract infection. Clinicians could relate to the potential usefulness of POCTs based on past experience. Existing POCTs were mostly used within consultation rooms. Some prescribers though were unfamiliar with the term 'POCT' test, preferring the term 'rapid diagnostic test'. Many clinicians were unfamiliar with POCTs for common infections, commonly used in some HICs to support clinical decision making for example, CRP POCT.

Some questioned the diagnostic accuracy of existing POCTs when POCT results did not corroborate their clinical findings. Some clinicians also perceived that existing POCTs had not lived up to their expectation by taking up precious time, or being difficult to implement in their primary care clinics.

However, most clinicians were receptive to the potential of new POCTs for common infections: to aid diagnosis; to provide an indication for patients that antibiotics are not needed; to guide treatment decisions (especially in the context of HIV/TB); to initiate treatment earlier; to improve prescribing quality; to give nurses additional tools before referring to a doctor/senior colleague; and to provide clinicians 'peace of mind' when withholding an antibiotic. One clinician viewed new POCTs as a potential 'game changer'.

[The POCT] will tell me exactly, must I give antibiotics or not. It will tell me I need to advise this person to use home remedies immediately or $[\ldots]$ manage them with antibiotics. It will save time and [reduce] unnecessary antibiotics. (P17, nurse)

Clinicians perceived that one of the major advantages of using a new POCT for common infections in their clinics might be to provide an important patient communication tool that antibiotics were unlikely to be of benefit.

...Especially with patients that pressure you for antibiotics, you have something to show them that [antibiotics] aren't really indicated. (P9, nurse)

This in turn may support non-antibiotic management strategies, limit potentially harmful antibiotic exposure and give an indication for patients of the likely duration of illness and when they could return to work.

I think they will add tremendous value in terms of what you can tell your patient. You know what sort of education you can do. What safety netting you can do. (P4, doctor)

Clinicians reported that new POCTs for common infections might improve the relationship between patient and clinician, give patients more confidence about the diagnosis, and might appease patients' expectations for antibiotics-especially patients who had low health literacy. ...if you do find the test is negative for an antibiotic, tell [the patient], 'it's not that [I'm] not believing [you], or 'you're not sick'. It's just we're going to treat you in a different way. (P19,nurse)

Yet, they also acknowledged that any new POCT would not replace clinical judgement and would be potentially most helpful for 'grey areas', where it was unclear whether patients were likely to benefit from antibiotics.

...with respiratory infections, I think there [are] a lot of grey areas. And I'd need to be convinced that POCT is going to reduce that grey area significantly. (P13,doctor)

Some clinicians, however, were apprehensive about new POCTs: their (imperfect) diagnostic accuracy; their inability to confidently rule out a secondary bacterial infection developing; and that use of tests alone could never take into account important social determinants of prescribing. They were concerned that new POCTs would disrupt clinician-patient communication, replace or precede the clinical examination, and encourage reliance on POCTs to make a diagnosis. Some clinicians perceived that patients would expect POCTs at every consultation and might encourage unnecessary consultation. However, not all clinicians shared this opinion.

No, I don't think [patients will re-consult]. Patients come when they're sick and they know they're going to wait a long time to be seen, so patients usually come if there is a good reason to come. (P23, doctor)

Clinicians identified two main barriers that would need to be overcome before introducing any new POCT in their workplace. Their first concern related to resourcing and cost effectiveness. Currently prescribed antibiotics are much cheaper than any POCT currently available and so arguments for commission based on cost alone were felt unlikely to be persuasive. However, new POCTs might reduce unnecessary antibiotics as well as special investigations for common infections, decrease laboratory transport costs and unnecessary referrals to hospitals, improve health service delivery, and potentially reduce antibiotic resistance.

I think [POCTs] will outweigh [the costs] especially because of where we are. We cannot afford to be prescribing antibiotics where they are not needed. (P3, doctor)

Second, clinicians were concerned that the perceived workload of performing and maintaining POCTs would discourage continued use. This concern stemmed from experience with existing POCTs where reagents and supplies often ran out or went missing, POCT machines were often not user-friendly, or POCT kits would be frequently changed requiring training updates. However, other clinicians perceived that new POCTs would not have a detrimental impact on staff workload provided 
that there was buy-in from clinicians and ongoing staff training.

\section{DISCUSSION}

\section{Summary of main findings}

Clinicians working in the LMIC context of publicly funded clinics in the Western Cape Metro of South Africa reported recognising the value of existing clinic POCTs but acknowledged that these POCTs had not always lived up to their expectations. They were generally positive about the possible introduction of new POCTs to support decision-making for common infections, for improving patient outcomes and reducing unnecessary antibiotic prescriptions and further investigations. Most clinicians perceived that new POCTs might support patient communication to more often achieve evidence-based prescribing decisions.

Clinicians were clear that any new POCTs should not replace a thorough history and physical examination when managing common infections. Some clinicians voiced their concerns about diagnostic accuracy and the lack of prognostic value of a POCT result. They cited resource limitations and cost-effectiveness considerations, and disruption to existing care provision as the main hurdles to overcome before adopting any new POCT into routine care.

\section{Comparison with existing research findings}

Our findings agree with studies from HIC primary care exploring benefits of POCT, ${ }^{38}$ where clinicians reported that POCTs reduced diagnostic uncertainty, improved targeting of treatment, and enhanced clinician-patient communication and relationships. ${ }^{35} 364344$ However, clinicians in HICs also expressed similar concerns to informants in our study in an LMIC setting about: test accuracy $^{3545}$; the additional time to use POCTs; the overreliance on tests; the undermining of clinical skills ${ }^{364}$; cost; the disruption to clinical workflow ${ }^{3544} 45$ and limited usefulness. ${ }^{35} 43$

Our findings are also similar to findings in qualitative studies set in other LMICs, in particular southeast Asia, that highlight an overall enthusiasm among healthcare workers with the introduction of POCTs, ${ }^{26}$ but also echo concerns about the impact on workload and constraints of available alternatives to antibiotic treatment. ${ }^{28}$ Clinicians in southeast Asia reported using a CRP POCT to support their negotiations with patients for a nonantibiotic strategy, helping to manage perceived patient expectations for antibiotics. However, these POCTs were also used to deal with explicit patient demands for antibiotics in the context of key context specific social determinants of health. ${ }^{26}{ }^{30}$ This is in contrast to a study set in six European countries, which found that clinicians and patients reported differing views on the use of a CRP POCT. Clinicians considered that the POCT was useful in achieving patient acceptance of a non-antibiotic strategy, while patients often thought the POCT was unnecessary if clinicians took the time to explain their decision. ${ }^{46}$ Clinicians in our study strongly perceived that new POCTs would support more effective clinician-patient communication about the benefit and harms of antibiotics.

None of our participants mentioned POCTs as a means to rule out serious infections ${ }^{36}$ nor that POCTs would enable better access to alternative diagnostic methods where laboratory access was inadequate. ${ }^{39} 40$ Clinicians in our study were less convinced about whether POCTs would provide more efficient care and fewer consultations. This is in contrast to European studies where clinicians felt that POCTs might lead to fewer reconsultations for the same or future illness episodes. ${ }^{43} 44$

\section{Strengths and limitations}

This qualitative study with clinicians working in the Western Cape Metro of South Africa, exploring their perceptions of making antibiotic prescribing decisions and the potential benefits and barriers of new POCTs for two common infection syndromes. We specifically focused on primary care clinicians working in publicly funded clinics as this is where most antibiotic prescribing occurs. We included both nurse and doctor prescribers from a range of clinics to reflect the breadth of antibiotic prescribing practice in this region and because many patients are managed by nurses in this setting. The main themes and subthemes were not noticeably different between participants in terms of profession, gender, race or by clinic setting.

We accept that our study has several limitations. Although we invited clinicians from a variety of primary care settings, our sampling was not purposive due to the time constraints of the study. Our convenience sampling may have introduced selection bias in favour of prescribers who were interested in POCTs. However, our findings also suggest that many clinicians were not familiar with existing POCTs for common infections used in HICs (eg, POCT CRP). In a few interviews, we had to explain what a POCT was which may have led to a one-sided description of the POCT and the potential for bias.

Some interview questions relate to the abstract concept of a new (hypothetical) POCT which clinicians had not used or heard of, rather than relating to actual experiences of using new POCTs. ${ }^{43}$ However, to limit ambiguity, we were careful to separate questions about existing POCTs and those that were hypothetical. Views of actually new POCTs may be entirely different if introduced, and indeed this has been shown in previous work where clinicians changed their views after having used a particular test. ${ }^{36}{ }^{43}$ Yet, identifying and anticipating clinicians' perceptions of barriers and opportunities of introducing new tests ahead of assessing their usefulness can be very useful to ensure maximum, minimally disruptive uptake for patient benefit.

We acknowledge that this study reflects the perceptions of clinicians, rather than the actual interaction between patient and clinician. Therefore, the findings need to be interpreted in this context. Anthropological and 
sociological research on POCTs for malaria in LMICs in Africa and Asia has found that the introduction of new POCTs into an established system of healthcare practices can lead to unforeseen implementation challenges and consequences. ${ }^{47}$ Therefore, POCTs for other conditions may face different implementation challenges and findings from one setting about specific infectious syndromes may not apply to other settings. Indeed, our findings may not be transferable to other parts of South Africa, or other LMIC analogous healthcare settings.

\section{Implications for clinical practice, policy and future research}

The use of POCTs for common infections in such resourcepoor settings may only affect a proportion of unnecessary antibiotic prescribing because there are important social determinants of prescribing in South Africa that seem to override guideline-driven prescribing. Multifaceted interventions which target multiple influences on clinician prescribing behaviour are therefore likely to be needed to improve patient outcomes while minimising risk from different patterns of antibiotic prescribing.

Further studies are needed in LMIC primary care settings to test the clinical and cost-effectiveness of POCTs for common infections. The interventions will need to take the perspectives of clinicians and patients into account for them to achieve maximum benefit. POCTs for common infections may not necessarily lead to better patient education about self-limiting infections unless clinicians are prepared to discuss their clinical findings and involve the patient in making decisions about treatment. Future trials adopting POCTs for common infection syndromes need to consider the way in which care is organised and fulfil the needs of the local context.

In conclusion, clinicians in this LMIC setting viewed POCTs as potentially useful for effectively addressing both clinical and social factors that influence their antibiotic prescribing decisions. They added that the introduction of new POCTs should be based on careful consideration of the best use of resources, which include a broader view of cost-effectiveness, and how POCTs might fit into existing patient workflows with minimum disruption.

Contributors $\mathrm{OvH}$ had full access to all of the data in the study and takes responsibility for the integrity of the data and the accuracy of the data analysis. Concept and design: OvH, MM, CB and ST-C. Drafting of the manuscript: OvH, MM, CB and ST-C. Qualitative analysis: OvH and ST-C. Administrative, technical or material support: MM, ST-C and CB is guarantor. All authors contributed to and approved the final manuscript. The corresponding author attests that all listed authors meet authorship criteria and that no others meeting the criteria have been omitted.

Funding The PETRI Study is funded by a Newton Fund/RCUK partnership scheme (NRF grant reference number (UID): 98556). This work is based on the research supported by the National Research Foundation. ST-C and CB received funding from the National Institute for Health Research Health Protection Research Unit (NIHR HPRU) in Healthcare Associated Infections and Antimicrobial Resistance at the University of Oxford in partnership with Public Health England (PHE) (HPRU-201210041). CB is a NIHR Senior Investigator, and Clinical Director of the University of Oxford Primary Care and Vaccines Clinical Trials Collaboration, and the NIHR Oxford Community Medical technology and Invitro diagnostics $\mathrm{Co}$-operative. CB is funded/ received by National Institute for Health Research (NIHR) Community Healthcare Medtech and in Vitro Diagnostics Co-operative at Oxford health NHS Foundation Trust.
Disclaimer The views expressed are those of the author(s) and not necessarily those of the NHS, the NIHR, the Department of Health and Social Care or Public Health England. The views expressed are those of the author and necessarily those of the NHS, the NIHR or the Department of Health and Social Care.

Competing interests All authors have completed the ICMJE uniform disclosure form at www.icmje.org/coi_disclosure.pdf and declare: support from the NIHR for the submitted work; no financial relationships with any organisations that might have an interest in the submitted work in the previous three years; no other relationships or activities that could appear to have influenced the submitted work.

\section{Patient consent for publication Not required.}

Ethics approval Ethical approval was obtained from University of Oxford (OxTREC Ref: 508-18) and University of cape Town (HREC 071/2018).

Provenance and peer review Not commissioned; externally peer reviewed.

Data availability statement Data are available on reasonable request.

Open access This is an open access article distributed in accordance with the Creative Commons Attribution Non Commercial (CC BY-NC 4.0) license, which permits others to distribute, remix, adapt, build upon this work non-commercially, and license their derivative works on different terms, provided the original work is properly cited, appropriate credit is given, any changes made indicated, and the use is non-commercial. See: http://creativecommons.org/licenses/by-nc/4.0/.

\section{REFERENCES}

1. Costelloe C, Metcalfe C, Lovering A, et al. Effect of antibiotic prescribing in primary care on antimicrobial resistance in individual patients: systematic review and meta-analysis. BMJ 2010;340:c2096.

2. van Hecke $\mathrm{O}$, Wang $\mathrm{K}$, Lee $\mathrm{JJ}$, et al. Implications of antibiotic resistance for patients' recovery from common infections in the community: a systematic review and meta-analysis. Clin Infect Dis 2017;65:371-82.

3. Kenealy T, Arroll B, Cochrane Acute Respiratory Infections Group. Antibiotics for the common cold and acute purulent rhinitis. Cochrane Database Syst Rev 2013;51.

4. Spinks A, Glasziou PP, Del Mar CB, et al. Antibiotics for sore throat. Cochrane Database Syst Rev 2013;139.

5. Spurling GKP, Del Mar CB, Dooley L, et al. Delayed antibiotics for respiratory infections. Cochrane Database Syst Rev 2013.

6. Venekamp RP, Sanders SL, Glasziou PP, et al. Antibiotics for acute otitis media in children. Cochrane Database Syst Rev 2015;303.

7. Center for Disease Dynamics Economics and Policy. State of the World's Antibiotics. Washington DC: CEEP, 2015.

8. Pillay-van Wyk V, Msemburi W, Laubscher R, et al. Mortality trends and differentials in South Africa from 1997 to 2012: second National burden of disease study. Lancet Glob Health 2016;4:e642-53.

9. National Department of Health South Africa. Essential drugs programme. standard treatment guidelines and essential medicines list for South Africa, primary healthcare level, 2018.

10. Mendelson M MP. The South African antimicrobial resistance strategy framework: AMR control, 2015.

11. Gasson J, Blockman M, Willems B. Antibiotic prescribing practice and adherence to guidelines in primary care in the Cape town Metro district, South Africa. S Afr Med J 2018;108:304-10.

12. Hoffman D, Botha J. An assessment of factors influencing the prescribing of antibiotics in acute respiratory illness: a questionnaire study. S Afr Fam Pract 2003;45:20-4.

13. Farley E, Stewart A, Davies M-A, et al. Antibiotic use and resistance: knowledge, attitudes and perceptions among primary care prescribers in South Africa. S Afr Med J 2018;108:763-71.

14. National Institute for Health and Clinical Excellence. Respiratory Tract Infections - Antibiotic Prescribing: Prescribing of Antibiotics for SelfLimiting Respiratory Tract Infections in Adults and Children in Primary Care. London: National Institute for Health and Clinical Excellence (UK), 2008.

15. Jones CHD, Glogowska M, Locock L, et al. Embedding new technologies in practice - a normalization process theory study of point of care testing. BMC Health Serv Res 2016;16:591.

16. Horwood J, Cabral C, Hay AD, et al. Primary care clinician antibiotic prescribing decisions in consultations for children with RTIs: a qualitative interview study. Br J Gen Pract 2016;66:e207-13.

17. McNulty CAM, Nichols T, French DP, et al. Expectations for consultations and antibiotics for respiratory tract infection in primary care: the RTI clinical iceberg. Br J Gen Pract 2013;63:e429-36.

18. Fletcher-Lartey S, Yee M, Gaarslev C, et al. Why do general practitioners prescribe antibiotics for upper respiratory tract 
infections to meet patient expectations: a mixed methods study. BMJ Open 2016;6:e012244.

19. Lucas PJ, Cabral C, Hay AD, et al. A systematic review of parent and clinician views and perceptions that influence prescribing decisions in relation to acute childhood infections in primary care. Scand $J$ Prim Health Care 2015;33:11-20.

20. Brookes-Howell L, Hood K, Cooper L, et al. Understanding variation in primary medical care: a nine-country qualitative study of clinicians' accounts of the non-clinical factors that shape antibiotic prescribing decisions for lower respiratory tract infection. BMJ Open 2012;2:e000796.

21. Tonkin-Crine S, Yardley L, Little P. Antibiotic prescribing for acute respiratory tract infections in primary care: a systematic review and meta-ethnography. J Antimicrob Chemother 2011;66:2215-23.

22. Cooke J, Butler $\mathrm{C}$, Hopstaken $\mathrm{R}$, et al. Narrative review of primary care point-of-care testing (POCT) and antibacterial use in respiratory tract infection (RTI). BMJ Open Respir Res 2015;2:e000086

23. Tonkin-Crine SK, Tan PS, van Hecke O, et al. Clinician-targeted interventions to influence antibiotic prescribing behaviour for acute respiratory infections in primary care: an overview of systematic reviews. Cochrane Database Syst Rev 2017;9:CD012252.

24. Mendelson M, Røttingen J-A, Gopinathan U, et al. Maximising access to achieve appropriate human antimicrobial use in lowincome and middle-income countries. The Lancet 2016;387:188-98.

25. Parkes-Ratanshi R, Kikonyogo R, Hsieh Y-H, et al. Point-OfCare diagnostics: needs of African health care workers and their role combating global antimicrobial resistance. Int J STD AIDS 2019;30:404-10.

26. Haenssgen MJ, Charoenboon N, Althaus T, et al. The social role of Creactive protein point-of-care testing to guide antibiotic prescription in northern Thailand. Soc Sci Med 2018;202:1-12.

27. Khine Zaw Y, Charoenboon N, Haenssgen MJ, et al. A Comparison of Patients' Local Conceptions of Illness and Medicines in the Context of C-Reactive Protein Biomarker Testing in Chiang Rai and Yangon. Am J Trop Med Hyg 2018;98:1661-70.

28. Haenssgen MJ, Charoenboon N, Do NTT, et al. How context can impact clinical trials: a multi-country qualitative case study comparison of diagnostic biomarker test interventions. Trials 2019;20:111.

29. Althaus T, Greer RC, Swe MMM, et al. Effect of point-of-care Creactive protein testing on antibiotic prescription in febrile patients attending primary care in Thailand and Myanmar: an open-label, randomised, controlled trial. Lancet Glob Health 2019;7:e119-31.

30. Do NTT, Ta NTD, Tran NTH, et al. Point-Of-Care C-reactive protein testing to reduce inappropriate use of antibiotics for non-severe acute respiratory infections in Vietnamese primary health care: a randomised controlled trial. Lancet Glob Health 2016;4:e633-41.

31. Touboul-Lundgren P, Jensen S, Drai J, et al. Identification of cultural determinants of antibiotic use cited in primary care in Europe: a mixed research synthesis study of integrated design "Culture is all around us". BMC Public Health 2015;15:908.

32. Africa SS. Census. Pretoria statistics South Africa 2012, 2011.

33. Department of Health SA. Cape Metro District Health PLan 2018/19 2020/21, 2018.

34. Hardy V, Thompson M, Keppel GA, et al. Qualitative study of primary care clinicians' views on point-of-care testing for C-reactive protein for acute respiratory tract infections in family medicine. BMJ Open 2017; 7:e012503.

35. Butler CC, Simpson S, Wood F. General practitioners' perceptions of introducing near-patient testing for common infections into routine primary care: A qualitative study. Scand J Prim Health Care 2008;26:17-21.

36. Cals JWL, Chappin FHF, Hopstaken RM, et al. C-Reactive protein point-of-care testing for lower respiratory tract infections: a qualitative evaluation of experiences by GPs. Fam Pract 2010;27:212-8.

37. Huddy JR, Ni MZ, Barlow J, et al. Point-Of-Care C reactive protein for the diagnosis of lower respiratory tract infection in NHS primary care: a qualitative study of barriers and facilitators to adoption. BMJ Open 2016;6:e009959.

38. Jones CHD, Howick J, Roberts NW, et al. Primary care clinicians' attitudes towards point-of-care blood testing: a systematic review of qualitative studies. BMC Fam Pract 2013;14:117.

39. Mashamba-Thompson T, Jama N, Sartorius B, et al. Implementation of point-of-care diagnostics in rural primary healthcare clinics in South Africa: perspectives of key stakeholders. Diagnostics 2017;7.

40. Rasti R, Nanjebe D, Karlström J, et al. Health care workers' perceptions of point-of-care testing in a low-income country-A qualitative study in southwestern Uganda. PLoS One 2017;12:e0182005

41. Braun V, Clarke V. Using thematic analysis in psychology. Qual Res Psychol 2006;3:77-101.

42. Glaser BG, Strauss AL. The discovery of Grounded theory: strategies for qualitative research. New York, NY: AldineDe Gruyter, 1967.

43. Cals JWL, Butler CC, Dinant G-J. 'Experience talks': physician prioritisation of contrasting interventions to optimise management of acute cough in general practice. Implementation Sci 2009;4.

44. Wood F, Brookes-Howell L, Hood K, et al. A multi-country qualitative study of clinicians' and patients' views on point of care tests for lower respiratory tract infection. Fam Pract 2011;28:661-9.

45. Gillam S, Freedman D, Naughton B, et al. An evaluation of near patient testing in general practice. J Eval Clin Pract 1998;4:165-9.

46. Tonkin-Crine S, Anthierens S, Hood K, et al. Discrepancies between qualitative and quantitative evaluation of randomised controlled trial results: achieving clarity through mixed methods triangulation. Implementation Sci 2015;11.

47. Beisel U, Umlauf R, Hutchinson E, et al. The complexities of simple technologies: re-imagining the role of rapid diagnostic tests in malaria control efforts. Malar J 2016;15:64. 